\title{
Class B Gene Expression and the Modified ABC Model in Nongrass Monocots
}

\author{
Akira Kanno*, Mutsumi Nakada, Yusuke Akita, and Masayo Hirai \\ Graduate School of Life Sciences, Tohoku University, Katahira 2-1-1, Aoba-ku, \\ Sendai 980-8577, Japan \\ E-mail: kanno@ige.tohoku.ac.jp
}

Received August 9, 2006; Revised December 28, 2006; Accepted January 4, 2007; Published February 19, 2007

The discovery of the MADS-box genes and the study of model plants such as Arabidopsis thaliana and Antirrhinum majus have greatly improved our understanding of the molecular mechanisms driving the diversity in floral development. The class B genes, which belong to the MADS-box gene family, are important regulators of the development of petals and stamens in flowering plants. Many nongrass monocot flowers have two whorls of petaloid organs, which are called tepals. To explain this floral morphology, the modified ABC model was proposed. This model was exemplified by the tulip, in which expansion and restriction of class $B$ gene expression is linked to the transition of floral morphologies in whorl 1. The expression patterns of class B genes from many monocot species nicely fit this model; however, those from some species, such as asparagus, do not. In this review, we summarize the relationship between class B gene expression and floral morphology in nongrass monocots, such as Liliales (Liliaceae) and Asparagales species, and discuss the applicability of the modified ABC model to monocot flowers.

KEY WORDS: modified ABC model, class B gene, tulip, lily, Agapanthus praecox, Muscari armeniacum, Tricyrtis affinis, asparagus, Phalaenopsis equestris, Crocus sativus, Dendrobium crumenatum

\section{INTRODUCTION}

Flower development in plants can be subdivided into several major steps, including floral induction, floral meristem formation, and floral organ development. Genetic control of the different steps of flower development is achieved by a hierarchy of interacting regulatory genes[1]. The identification of floral organ development as one of the steps in flower development was recognized during studies of homeotic mutants in which the identity of floral organs was changed. Genetic mutants of Arabidopsis thaliana and Antirrhinum majus were categorized into three classes (A, B, and C), and functional analyses of these mutants led to the ABC model of flower development[2]. Class A mutants have carpels in the first whorl instead of sepals, and stamens in the second whorl instead of petals. Class B mutants have sepals rather than petals in the second whorl and carpels rather than stamens in the third whorl. Class $\mathrm{C}$ mutants have petals instead of stamens in the third whorl and sepals instead of carpels in the fourth whorl, and an additional flower arises in the fourth whorl. Based on these classes of single mutants, and all combinations of double and triple mutants, the ABC model proposed that there are three functional classes of floral organ identity genes, called A, B, and C. The activity of class A genes (APETALA1 [AP1]

*Corresponding author. 
and APETALA2 [AP2] in A. thaliana, SQUAMOSA in A. majus) is required in the outer two whorls (first and second), class B genes (APETALA3 [AP3] and PISTILLATA [PI] in A. thaliana, DEFICIENS [DEF] and GLOBOSA [GLO] in A. majus) function in the second and third whorls, and class $\mathrm{C}$ genes (AGAMOUS $[A G]$ in A. thaliana, PLENA in A. majus) act in the inner two whorls (third and fourth). More recently, this model was extended to include class D genes, which specify the identity of ovules within the carpel[1,3,4]. An additional group of floral organ identity genes, the E-class genes (SEPALLATA [SEP],[5]), are involved in specifying petals, stamens, and carpels[6,7]. The model has therefore been renamed the ABCDE model of flower development. All genes involved in the ABCDE model, except for $A P 2$, belong to the family of MADS-box genes that encode MADS-box transcription factors[8].

\section{Modified ABC Model in Tulip}

In contrast to the flowers of higher eudicots, many nongrass monocot flowers, such as lily and tulip, have three outer tepals, three inner tepals, six stamens, and three carpels (Fig. 1A). It is difficult to fully account for this type of floral morphology using the classical ABC model. To explain this floral morphology, van Tunen et al. proposed a modified ABC model: the expression of class B genes was extended to whorl 1, with the result that the floral organs of the first and second whorls have almost the same petaloid structure (Fig. 1B, [9]). This model was based on the morphology of wild-type and mutant flowers of tulip (Tulipa gesneriana, Liliaceae). In a viridiflora tulip mutant, the organs of the outer two whorls are greenish and resemble the sepals of higher eudicots, and the six stamens are transformed into carpeloid organs. Another mutant flower has tepal-like structures in whorls 1, 2, and 3, and a new floral structure arises from the center of the flower. These phenotypes are similar to the phenotypes observed for class B and class C mutants of higher eudicots, respectively. Based on these morphological observations, van Tunen et al. proposed the modified ABC model, although they did not analyze these mutants using genetics or molecular biology.

Ten years after the modified ABC model was proposed, putative class $\mathrm{B}$ genes were isolated from tulip[10], including two DEF-like genes (TGDEFA, TGDEFB) and one GLO-like gene (TGGLO). The transcripts of all three genes are detected in floral organs and not in vegetative organs. TGDEFA and TGDEFB are strongly expressed in whorls 1,2 , and 3 , and TGGLO is strongly expressed in the outer three whorls and weakly in carpels (Fig. 1C). In A. thaliana and A. majus, both types of class B genes (DEF- and GLO-like genes) are needed for B function because their gene products function as heterodimers, and both $D E F$-like and GLO-like genes are expressed in outer and inner tepals and stamens in tulip. This expression pattern nicely fits the modified ABC model.

\section{The Expression of Class B Genes in Other Monocots}

\section{Liliaceae (Liliales)}

Several class B genes were isolated from lily, a liliaceous plant closely related to tulip. One $D E F$-like ( $L R D E F)$ and two GLO-like (LRGLOA and $L R G L O B$ ) genes has been isolated from Lilium regale (Fig. 2,[1,11]), and one DEF-like gene (LMADS1) has been isolated from L. longiflorum[12]. A Northern blot analysis of dissected floral organs showed that the $L R D E F$ gene is expressed in outer and inner tepals and stamens. This expression pattern, like that of tulip, supports the modified ABC model [1]. On the other hand, the expression pattern of LMADS1 is slightly different from that of LRDEF. A Northern blot analysis showed that LMADS1 is expressed strongly in whorls 2 and 3 and weakly in whorls 1 and 4, whereas its protein accumulates only in whorls 2 and 3[12]. These results suggest the possibility that the floral morphology of L. longiflorum, which has two whorls of petaloid tepals, cannot be explained by the 
A
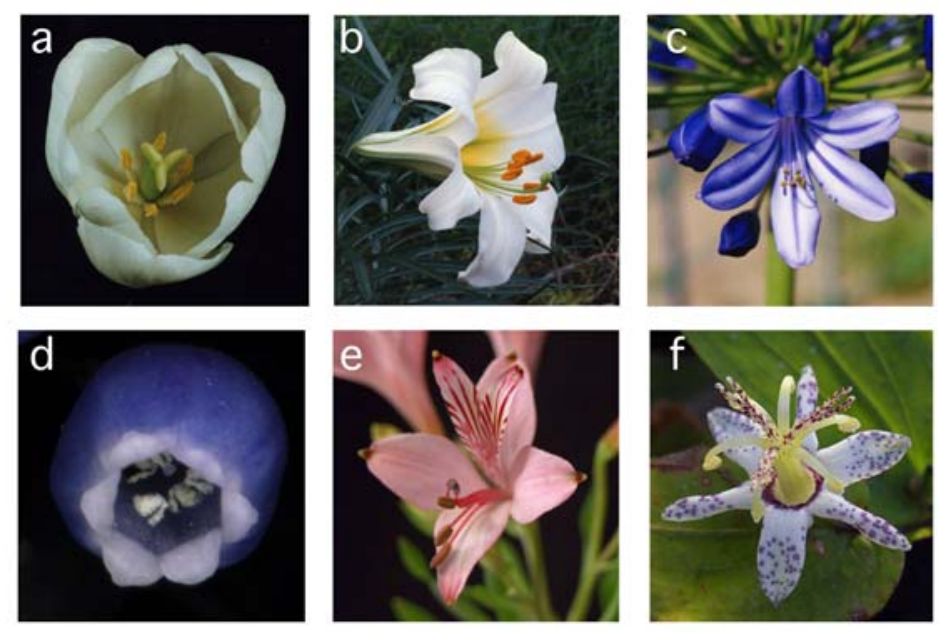

B
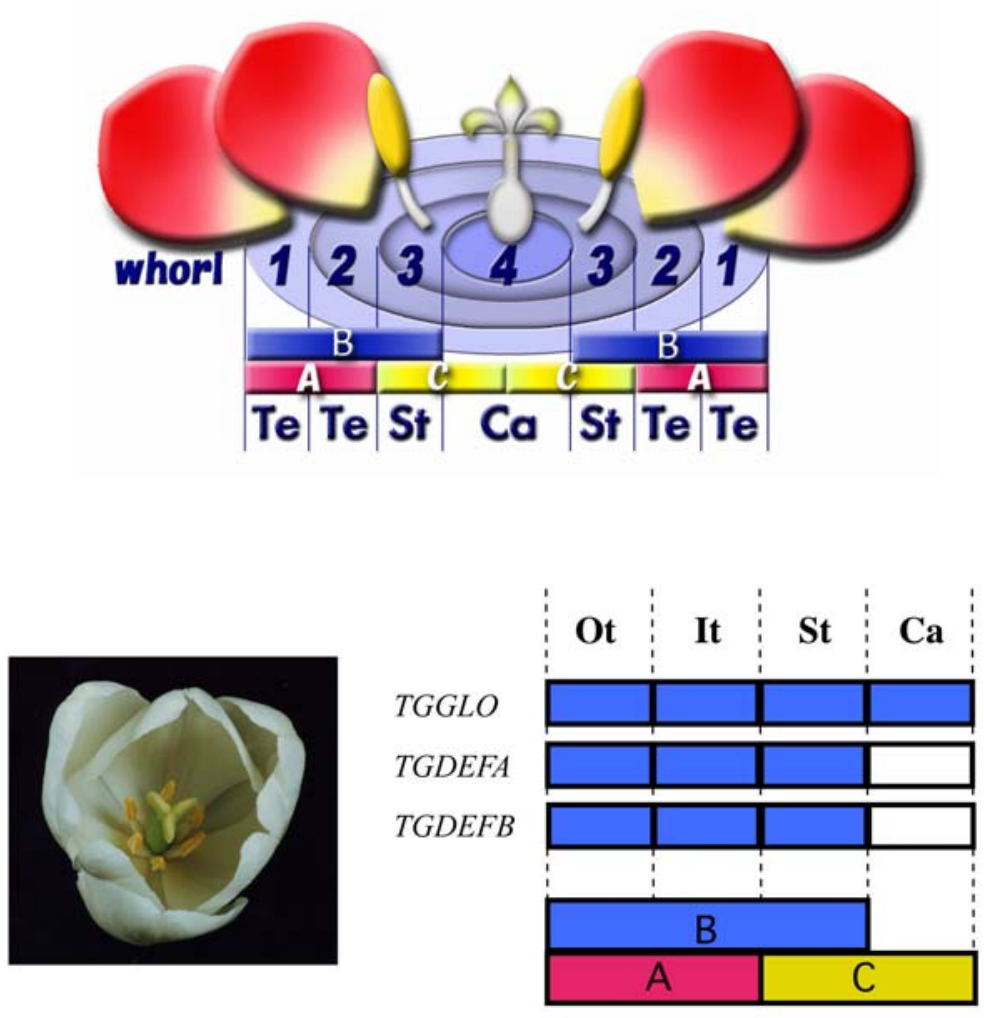

Figure 1. A. Monocot flowers with two whorls of petaloid organs. Tulipa gesneriana (a), Lilium regale (b), Agapanthus praecox (c), Muscari armeniacum (d), Alstroemeria ligtu (e), and Tricyrtis affinis (f). B. Modified ABC model proposed by van Tunen et al.[9]. This model explains the flower morphology of tulip: class B genes are expressed in whorl 1 as well as whorls 2 and 3, thus the organs of whorl 1 have the same petaloid character as those of whorl 2. C. The expression pattern of the class B genes (TGGLO, TGDEFA, and TGDEFB) from Tulipa gesneriana. Te, tepals; Ot, outer tepals; It, inner tepals; St, stamens; Ca, carpels. 


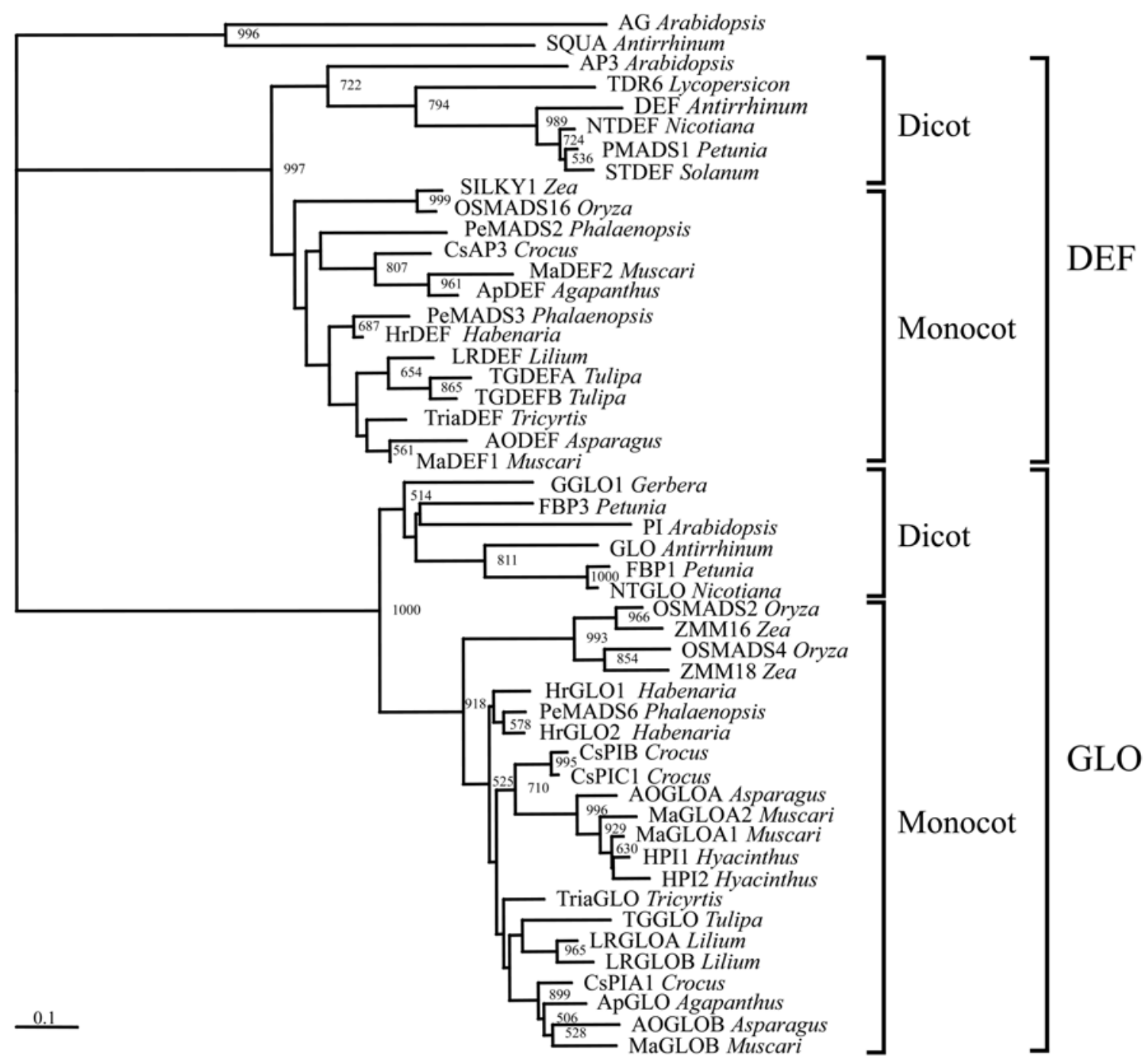

Figure 2. Phylogenetic tree of $D E F$ - and GLO-like genes. The neighbor-joining method was used. The numbers next to the nodes give bootstrap values from 1,000 replicates.

modified ABC model. However, these expression analyses with Northern hybridization and Western blotting were performed using dissected floral organs. In order to clarify these inconsistent results in the two lily species, the accumulation of the mRNA and protein of class B genes should be analyzed in floral organ primordia using flowers at very early stages. Given that multiple $D E F$-like genes exist in tulip, the possibility also remains that another lily $D E F$-like gene is expressed in the outer whorl of tepals.

A better functional understanding of $D E F$ - and GLO-like genes in Liliaceae plants would be obtained by transgenic approach, but the genetic transformation of lily and tulip is very difficult. Therefore, we have started to isolate class B genes from another Liliaceae plant, Tricyrtis affinis, because Agrobacterium-mediated transformation system has been recently developed in Tricyrtis species[13]. We have already isolated TriaDEF and TriaGLO genes from T. affinis (Fig. 2). An RT-PCR analysis showed that TriaDEF is expressed in whorls 1, 2, and 3, whereas TriaGLO is expressed in all floral organs (Fig. 3A: our unpublished result). These results in T. affinis are consistent with a modified ABC model. 


\section{Asparagales}

Like Liliaceae plants, most flowers in Asparagales have two whorls of petaloid tepals. Many class B genes have been isolated from several plants belonging to Asparagales, and their gene expression patterns have been analyzed. Most of the results support the modified ABC model.

Agapanthus praecox, which is a member of Asparagales, has two whorls of almost identical petaloid organs (Fig. 1A, panel c). ApDEF and ApGLO have been isolated from this plant (Fig. 2, [14]). An RTPCR analysis detected ApGLO expression in whorls 1, 2, and 3, whereas ApDEF transcripts were detected in all floral organs (Fig. 3B,[14]). The expression patterns of these genes were also analyzed by in situ hybridization, which showed that both of these genes are expressed in whorl 1 as well as in whorls 2 and 3 during the early stages of floral development in A. praecox (Fig. 3B:[14]). This is the first report to support the modified ABC model and to show that the class B genes are expressed in the floral organ primordia of the two outer whorls.

Muscari armeniacum, another member of Asparagales, has two whorls of petaloid tepals fused into a tube (Fig. 1A, panel d). One DEF-like gene, MaDEF1 (formerly MaDEF), has been isolated from this plant species[15]. An RT-PCR analysis using total RNA isolated from dissected floral organs by the laser microdissection system detected the MaDEF1 transcript in whorls 1, 2, and 3[15]. Later, an additional $D E F$-like gene (MaDEF2) and three GLO-like genes (MaGLOA1, MaGLOA2 and MaGLOB) were isolated from $M$. armeniacum (Fig. 2: our unpublished result). An RT-PCR analysis detected the transcripts of MaDEF2 and the three GLO-like genes in all floral organs (Fig. 4A: our unpublished result). The extended expression of class $B$ genes in whorl 1 fits the modified ABC model. The additional expression of class B genes in whorl 4, which is not consistent with this model, is discussed in next section, "Unsolved problems of the modified ABC model."

Recently, DEF-like (CsatAP3) and GLO-like (CsatPIc) genes were isolated from Crocus sativus[16]. An RT-PCR analysis detected the transcripts of both of these genes in all floral organs. This result of expanded class B gene expression in whorl 1 is similar to that found in $M$. armeniacum, supporting the modified ABC model.

The orchids (Orchidaceae) are a huge family within the order Asparagales whose species are distributed throughout the world and vary greatly in morphology. So far, class B genes have been isolated from three orchid species, Phalaenopsis equestris[17,18], Dendrobium crumenatum [19], and Oncidium Gower Ramsey[20], although no GLO-like gene of Oncidium has been reported. Four DEF-like genes, PeMADS2, PeMADS3, PeMADS4, and PeMADS5[17], and one GLO-like gene, PeMADS6[18], have been reported in $P$. equestris, one of the most important horticultural plants among orchids. The four DEF-like genes are differentially expressed. The transcripts of PeMADS2 and PeMADS5 are found in petaloid sepals, petals (including lips), and columns, but are not found in pollinia. PeMADS3 is expressed in petals, lips, and columns, whereas the expression of PeMADS4 is restricted to lips and columns. PeMADS6 transcripts are detected in $P$. equestris in the whorl 1 sepals and whorl 2 petals, as well as lips and columns (Fig. 3C). The expanded expression of DEF-like (PeMADS2 and PeMADS5) and GLO-like (PeMADS6) genes in whorl 1 supports the modified ABC model.

Several MADS-box genes containing class $B$ genes were reported in another orchid, $D$. crumenatum[19]. From D. crumenatum, two DEF-like genes (DcOAP3A and DcOAP3B) and one GLOlike gene $(D c O P I)$ were isolated and the localization of their mRNA was examined. DcOAP3A mRNA was detected in all floral organs and leaves, whereas DcOAP3B mRNA was found in petals and lips (whorl 2), anthers (whorl 3), and columns (whorl 4, excluded anthers and their caps). In contrast, DcOPI was expressed in all floral organs. These expression patterns were also confirmed by in situ hybridization. These results in D. crumenatum are reminiscent of those in C. sativus and M. armeniacum. 
A

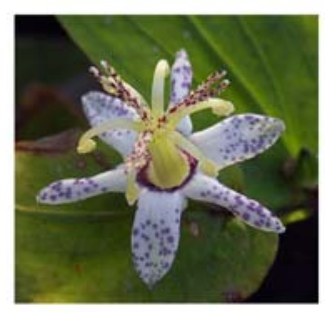

Tricyrtis

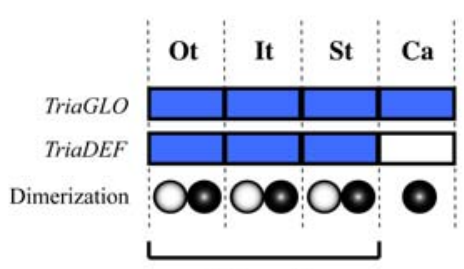

B function
B

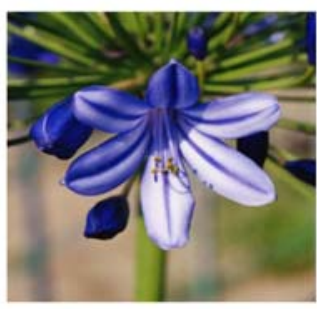

Agapanthus

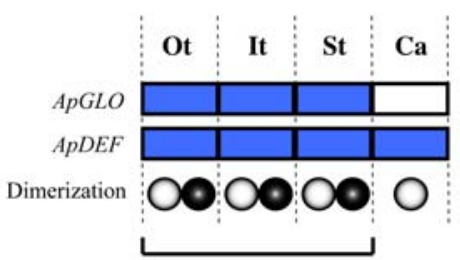

B function
C

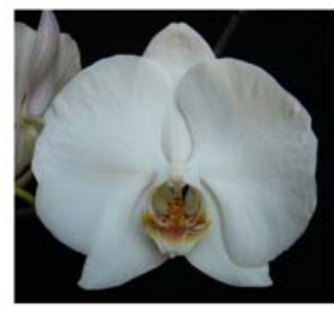

Phalaenopsis

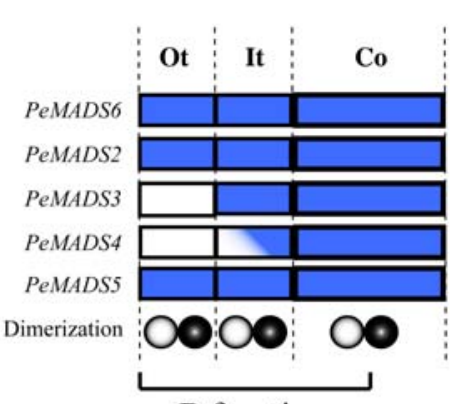

B function

Figure 3. The expression pattern of the class B genes from Tricyrtis affinis (A), Agapanthus praecox (B), and Phalaenopsis equestris (C). RT-PCR and/or Northern hybridization showing the organ-specific expression in each whorl. Shaded boxes indicate the expression of class $\mathrm{B}$ genes. The expression of $P$. equestris PeMADS4 is only observed in the lip and not in other inner tepals. These expression patterns of the class B genes support a modified ABC model. The dimerization pattern is shown below the expression patterns. Open and closed circles show DEF-like and GLO-like proteins, respectively. Ot, outer tepals; It, inner tepals; St, stamens; Ca, carpels; Co, columns.

Phylogenetic analysis showed that monophyly of monocot GLO-like genes is well supported (Fig. 2). It is interesting to note that the GLO-like genes from Asparagales are divided into two clusters in the phylogenetic tree: one cluster contains CsPIA1 (C. sativus), ApGLO (A. praecox), AOGLOB (Asparagus officinalis), and MaGLOB (M. armeniacum), and the other group contains CsPIB and CsPIC1 (C. sativus), AOGLOA (A. officinalis), MaGLOA1 and MaGLOA2 (M. armeniacum), and HPI1 and HPI2 (Hyacinthus orientalis). This indicates that both recent and ancient gene duplication events occurred during the evolution of Asparagales, and the ancient duplication that generated the GLO-like genes in these two clusters occurred before the divergence of these genera. $D E F$-like genes from Asparagales are also divided into two clusters: one includes MaDEF1 (M. armeniacum) and AODEF (A. officinalis), and the other contains MaDEF2 (M. armeniacum), ApDEF (A. praecox), and CsAP3 (C. sativus). This indicates that, as with the GLO-like genes, the gene duplication event in $D E F$-like genes occurred before the divergence of these genera. $D E F$-like genes from $M$. armeniacum fall into each cluster, but it is not clear whether two (or more) $D E F$-like genes might exist in other plants in Asparagales, such as $C$. sativus and $A$. praecox. 


\section{Unsolved Problems of The Modified ABC Model}

Many of the data on class B gene expression support the modified ABC model in Liliales (Liliaceae) and Asparagales, showing that petaloid tepals in monocots might correlate with the expansion of class B gene expression. However, there are some interesting results in monocot species that are not easy to explain.

\section{DEF- and GLO-Like Genes Are Not Expressed in Whorl 1 in Garden Asparagus}

Garden asparagus (Asparagus officinalis) has two whorls of almost identical petaloid tepals, similar to lily and tulip. One DEF-like (AODEF) and two GLO-like (AOGLOA, AOGLOB) genes have been isolated from asparagus, and in situ hybridization showed that the expression of these genes is restricted to whorls 2 and 3 (inner tepals and stamens, respectively); no transcripts are detected in whorl 1 (outer tepals; Fig. 4B)[21,22]. Since both $D E F$ - and GLO-like genes are required for the B-function in eudicots, it is likely that the B-function in asparagus is restricted to whorls 2 and 3 . Thus, the results of the asparagus class B gene expression do not support the modified ABC model, which proposes that two whorls of petaloid tepals are formed due to expanded class $\mathrm{B}$ gene expression in addition to class A gene expression. Is there no B-function in whorl 1 in asparagus? There are three hypotheses explaining the floral morphology in asparagus: (1) these class B genes are not involved in tepal development, (2) there are additional DEFand GLO-like class B genes that are specifically involved in outer tepal development, and (3) class B genes are transcribed in whorls 2 and 3 and the products move to whorl 1 (see[23]). In asparagus, one floral homeotic mutant is known in which the stamens are homeotically changed to carpels and the petaloid tepals are changed to sepaloid or leaf-like structures[23,24]. Because the mutant phenotype is very similar to that of the tulip "B mutant" reported previously[9], we favor the second hypothesis. There might be several class B genes in asparagus, but only three have already been isolated, and the regulatory mechanism of the expression of these class B genes may be damaged in the mutant; thus, the phenotype may arise from the lack of B function in whorls 1, 2, and 3. Further genetic and molecular biological analyses using this mutant are needed to clarify the mechanism of floral development in asparagus.

\section{Both DEF- and GLO-Like Genes Are Expressed in Whorl 4 in Dendrobium crumenatum, Crocus sativus, and Muscari armeniacum}

In the ABC model, B-function genes work with A-function genes to specify the development of petals and with C-function genes to specify the development of stamens. In A. thaliana, transcripts of PI are detectable in whorls 2, 3, and 4, whereas those of AP3 are found in whorls 1, 2, and 3[25,26]. In tobacco, GLO-like gene (NTGLO) transcripts are restricted to the second and third whorls, and $D E F$-like gene (NTDEF) transcripts are detectable in all floral organs[27]. This class $\mathrm{B}$ gene expression in tobacco is similar to that in A. majus[8]. The expression of $D E F$ - and GLO-like genes overlaps in the second and third whorls and their gene products perform B functions in these plants. To date, many DEF- and GLOlike genes have been isolated from various plant species. Expression studies (summarized in[28]) have shown that the overlapping expression of $D E F$ - and GLO-like genes is restricted to stamens and petals (including petaloid tepals). In monocots with two whorls of petaloid tepals, overlapping expression of $D E F$ - and GLO-like genes is found in outer and inner tepals and stamens in tulip (Fig. 1C), A. praecox, and T. affinis (Fig. 3A, B). In contrast to these results, the additional expression of both DEF- and GLOlike genes in whorl 4 has been reported in 2 species. 
A

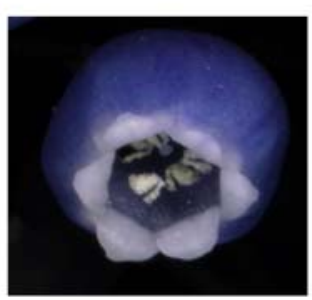

Muscari

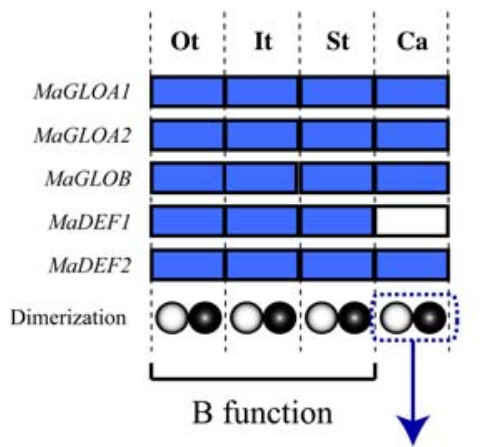

B function?
B

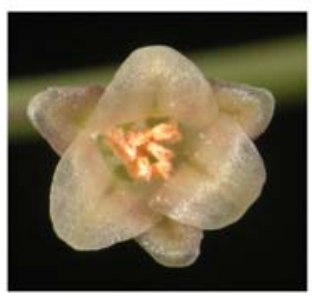

Asparagus

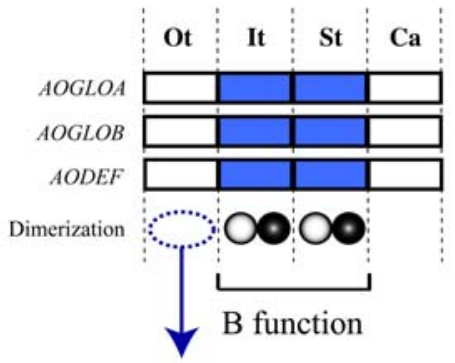

No B function?
C

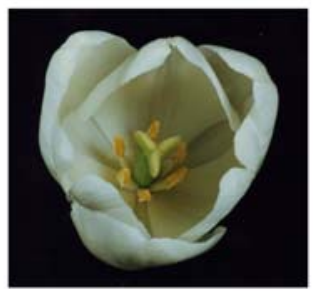

Tulipa

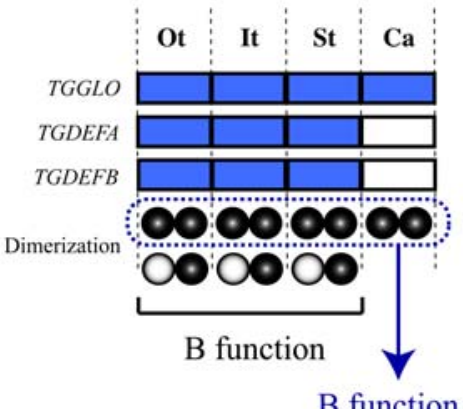

Figure 4. The expression pattern of the class B genes from Muscari armeniacum (A), Asparagus officinalis (B), and Tulipa gesneriana (C). Shaded boxes indicate the expression of class B genes. The expression patterns of some of these genes indicate that the modified ABC model is not strictly applicable to these monocots. The dimerization patterns are shown below the expression patterns. Open and closed circles show DEFlike and GLO-like proteins, respectively. Ot, outer tepals; It, inner tepals; St, stamens; Ca, carpels.

Recently, three B-function genes, DcOAP3A, DcOAP3B, and DcOPI, were isolated from the orchid D. crumenatum[19]. DcOAP3A and DcOPI are expressed in all floral organs, including petaloid sepals, petals, lips, and male and female reproductive organs, whereas DcOAP3B is expressed in the second whorl of petals and lips and in the reproductive organs including pollinia and columns. All three genes, $D c O A P 3 A, D c O A P 3 B$, and DcOPI, are expressed in the lower part of the column, which is equivalent to whorl 4 (carpels) in A. thaliana. This expanded expression of class B genes in whorl 4 was also detected in crocus[16]. An RT-PCR expression analysis indicated that CsatAP3 and CsatPIc (DEF- and GLO-like genes, respectively, from crocus) are expressed in all of four floral organs. Moreover, an RT-PCR analysis in M. armeniacum detected the transcripts of three GLO-like genes (MaGLOA1, MaGLOA2, and $M a G L O B)$ in all floral organs, and one $D E F$-like gene (MaDEF2) was expressed in whorl 4 as well as whorls 1, 2, and 3 (Fig. 4A: our unpublished result). The extended expression of class B genes into whorl 1 fits the modified ABC model; however, the additional expression in whorl 4 is not consistent with this model. Is there B-function in whorl 4 in these plants? There are two possibilities to explain this result. One possibility is that DEF- and GLO-like gene expression is regulated post-transcriptionally, and the proteins are not synthesized in whorl 4. In L. longiflorum, the mRNA of a DEF-like gene (LMADS1) was detected strongly in whorls 2 and 3, and also weakly in whorls 1 and 4, whereas the LMADS1 protein was only detected in whorls 2 and 3, suggesting that the expression of LMADS1 is regulated posttranscriptionally[12]. Thus, it would be very interesting to analyze protein expression in whorl 4 in $D$. crumenatum, C. sativus, and M. armeniacum. Recent publications indicate that microRNAs can play regulatory roles in animals and plants by targeting mRNAs for cleavage or translational repression[29]. Therefore, microRNAs may act as negative regulators of class B gene expression, although direct regulation of MADS-box genes by microRNAs has not been reported yet. The other possibility is that the 
DEF/GLO-heterodimer of these species needs additional factor(s), which are not expressed in whorl 4, to have B-function in these plants. Based on the floral quartet model [30], the AP3/PI heterodimer has multimeric protein interactions with AP1, SEP, and AG in A. thaliana. In D. crumenatum, yeast twohybrid analyses have shown that the DEF-like proteins DcOAP3A and DcOAP3B form heterodimers with a GLO-like protein, DcOPI. Moreover, these DEF/GLO heterodimers interact with a SEP-like protein, DcOSEP, to form higher protein complexes. The transcripts of these genes, including DcOSEP, were detected in all floral organs, indicating that these products could form multimeric complexes in all four whorls. If there are factors that interact with $D$. crumenatum DEF/GLO-heterodimer, they would likely be MADS-box proteins like DcOSEP, although there is no evidence of these additional factors.

\section{Homodimerization of GLO-Like Proteins in Lily and Tulip}

In A. thaliana and A. majus, B function is provided by heterodimers of DEF- and GLO-like proteins. In tulip, gel retardation assays showed that two DEF-like proteins (TGDEFA and TGDEFB) cannot homodimerize, but instead form heterodimers with GLO-like proteins (TGGLO), as do eudicot DEF- and GLO-like proteins (Fig. 4C,[10]). The TGGLO gene in tulip is expressed in all floral organs, including carpels. Two DEF-like genes are expressed together with the TGGLO gene in whorls 1,2 , and 3, so that the DEF/GLO heterodimer would provide B function in these organs. However, it is very interesting that TGGLO can bind to the CArG-box transcription factor binding site as a homodimer. Therefore, TGGLO homodimer would exist in whorl 4 in tulip. Homodimerization of GLO-like proteins have also been reported in a lily, Lilium regale (LRGLOA and LRGLOB,[11]), although the expression of lily GLO-like genes has not been analyzed. Do these homodimers have B function or another function that is not known in $A$. thaliana or A. majus? LMADS1 (a DEF-like protein from L. longiflorum) can also form a homodimer, which was identified in a yeast two-hybrid assay[12]. Although the expression of LMADS1 is restricted to whorls 2 and 3, the function of the LMADS1 homodimer also should be clarified.

\section{SUMMARY AND PERSPECTIVES}

Many monocot flowers have petaloid perianths in whorls 1 and 2, and it is difficult to fully account for this type of floral morphology using the classical ABC model. On the basis of morphological analyses of tulip mutants, van Tunen et al.[9] hypothesized that the formation of tepals is due to the expanded expression of class B genes into whorl 1. Moreover, a number of studies in nongrass monocots, such as tulip[10], P. equestris[17, 18], A. praecox[14], M. armeniacum[15], and D. crumenatum[19], lend support to a simple modification of the ABC model, the so-called modified ABC model[9]. In addition to being expressed in monocots, the AP3 and PI class B gene homologues are expressed in petaloid sepals in the first whorl in two dicots, Aquilegia alpina and A. clematiflora of Ranunculaceae[31]. The expansion of class $\mathrm{B}$ gene expression into the first whorl is consistent with the floral morphology of these species. Considering that the data are similar in monocots and dicots, the morphologies of whorls 1 and 2 may be generally linked to the expanded expression of class B genes. However, this does not apply in garden asparagus. In asparagus, the expression of class B genes is restricted to whorls 2 and 3, despite the presence of almost identical petaloid perianths in whorls 1 and 2[21,22]. In D. crumenatum, C. sativus, and $M$. armeniacum, the class B genes are expressed in whorls 1, 2, and 3, which fits the modified ABC model, but are also expressed in whorl 4, which does not fit the modified ABC model. However, the protein localization of the class B gene products is still unclear. 
A
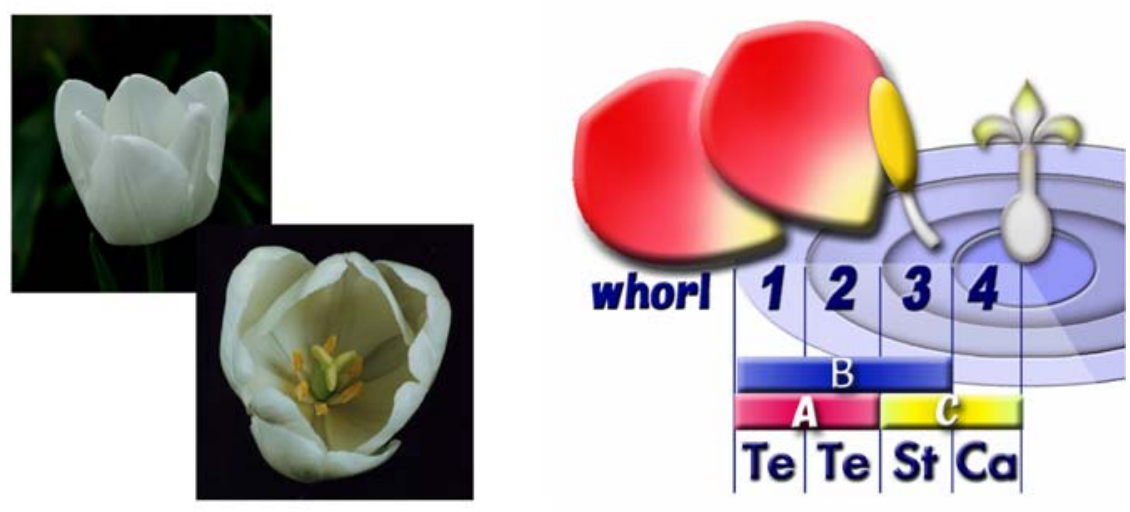

$\mathrm{Te}|\mathrm{Te}| \mathrm{St} \mathbf{C a}$

B
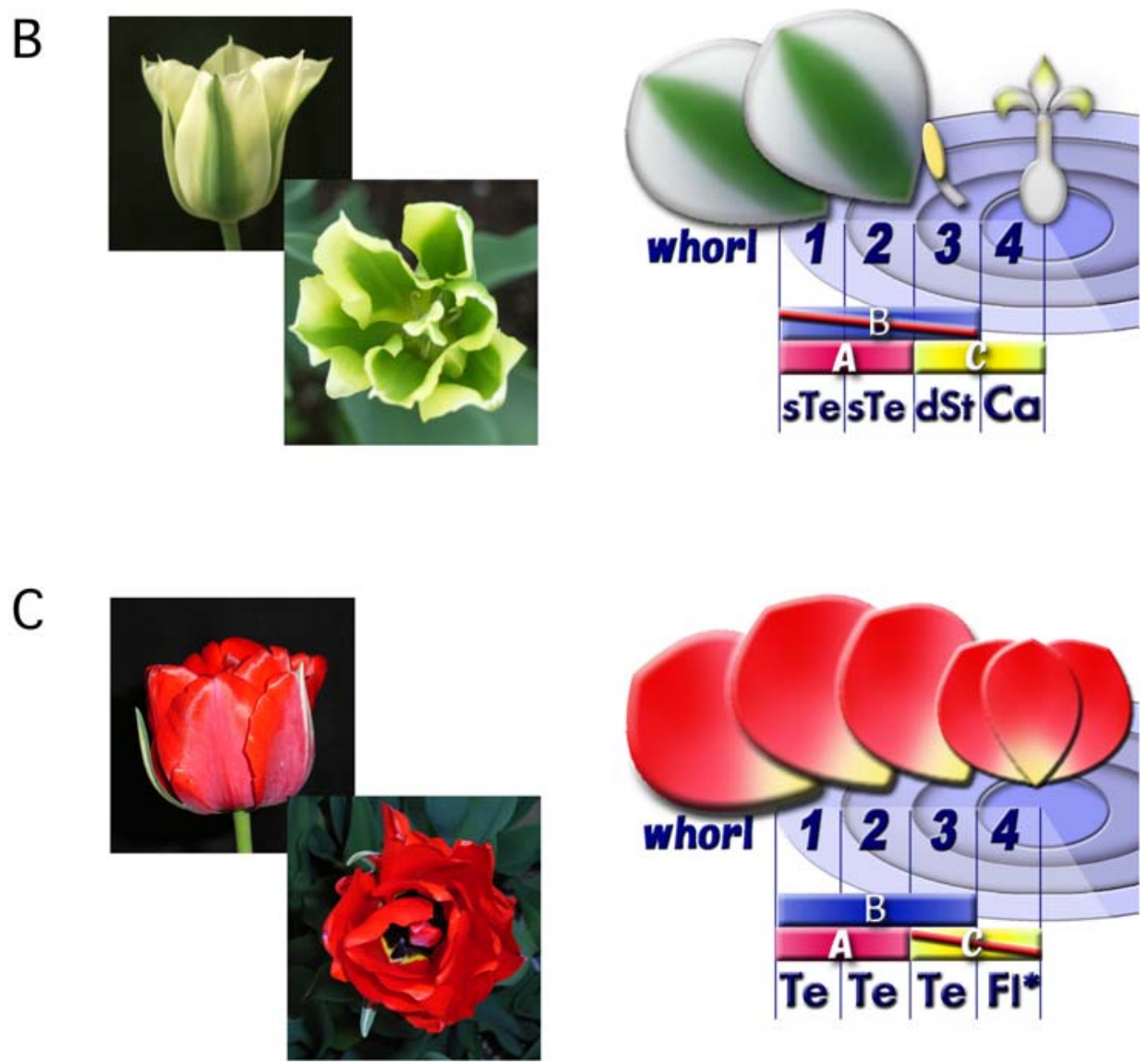

Figure 5. Various tulip flowers (left) and the models explaining these flower morphologies (right). A. The wild-type tulip flower has two whorls of petaloid tepals, stamens, and carpels. B. The viridiflora tulip flower has two whorls of partially greenish tepals (sepaloid), degenerated stamens, and carpels. This floral morphology is explained by reduced expression of class B gene(s). C. The double-flowered tulip has petaloid tepals in whorl 3 in addition to the outer two whorls and a new flower in the innermost whorl. This floral morphology is explained by reduced expression of the class C gene in whorls 3 and 4. Te, tepals; St, stamens; Ca, carpels; sTe, sepaloid tepals; dSt, degenerated stamens; Fl*, new flower. 
In order to clarify the molecular mechanism of petaloid tepal development in nongrass monocots, such as Liliales and Asparagales, functional studies with mutant analyses and genetic transformation are needed. Many of the species mentioned in this review are ornamental plants, and some known ornamental varieties are shown in figure 5. The wild-type tulip has two outer whorls of almost identical petaloid organs (Fig. 5A), whereas the viridiflora tulip has greenish ('sepaloid') tepals in whorls 1 and 2 and slightly degenerated stamens in whorl 3 that can be explained in the modified ABC model by a loss of B function (Fig. 5B). The double-flowered tulip has petaloid tepals in whorl 3 and the innermost whorl has become a new flower, which can be explained by a loss of $C$ function (Fig. 5C). The genetic analysis of these tulip mutants is difficult because it takes several years to get seeds of next generation, and the expression patterns of floral homeotic genes in these mutants are therefore very helpful for clarifying the mechanism of floral organ development in these plants. Recently, a system for producing transgenic plants has been developed for M. armeniacum, A. praecox, and Tricyrtis hirta via Agrobacteriummediated transformation[13,32,33]. Functional studies using this transformation technique will help illuminate the mechanisms of petaloid organ differentiation in monocots with two whorls of petaloid tepals.

\section{ACKNOWLEDGMENTS}

We deeply thank Dr. Masaru Nakano (Niigata University, Japan), Dr. Yoichiro Hoshino (Hokkaido University, Japan), and all of our lab members for helpful comments and discussion. This work was partly supported by a Grant-in-Aid for Scientific Research from the Ministry of Education, Culture, Sports, Science, and Technology of Japan.

\section{REFERENCES}

1. Theissen, G., Becker, A., Di Rosa, A., Kanno, A., Kim, J.T., Münster, T., Winter, K.-U. and Saedler, H. (2000) A short history of MADS-box genes in plants. Plant Mol. Biol. 42, 115-149.

2. Coen, E.S. and Meyerowitz, E.M. (1991) The war of the whorl: genetic interactions controlling flower development. Nature 353, 31-37.

3. Colombo, L., Franken, J., Koetje, E., van Went, J., Dons, H.J., Angenent, G.C., and van Tunen, A.J. (1995) The Petunia MADS-box gene FBP11 determines ovule identity. Plant Cell 7, 1859-1868.

4. $\quad$ Angenent, G.C. and Colombo, L. (1996) Molecular control of ovule development. Trends. Plant Sci. 1, 228232.

5. Theissen, G. (2001) Development of floral organ identity: stories from the MADS house. Curr. Opin. Plant Biol. 4, 75-85.

6. Pelaz, S., Ditta, G.S., Baumann, E., Wisman, E., and Yanofsky, M.F. (2000) B and C floral organ identity functions require SEPALLATA MADS-box genes. Nature 405, 200-203.

7. Honma, T. and Goto, K. (2001) Complexes of MADS-box proteins are sufficient to convert leaves into floral organs. Nature 409, 525-529.

8. Schwarz-Sommer, Z., Huijser, P., Nacken, W., Saedler, H., and Sommer, H. (1990) Genetic control of flower development by homeotic genes in Antirrhinum majus. Science 250, 931-936.

9. van Tunen, A.J., Eikeboom, W., and Angenent, G.C. (1993) Floral organogenesis in Tulipa. Flow. Newsl. 16, 33-38.

10. Kanno, A., Saeki H., Kameya T., Saedler H., and Theissen G. (2003) Heterotopic expression of class B floral homeotic genes supports a modified ABC model for tulip (Tulipa gesneriana). Plant Mol. Biol. 52, 831-841.

11. Winter, K.U., Weiser, C., Kaufmann, K., Bohne, A., Kirchner, C., Kanno, A., Saedler, H., and Theissen, G. (2002) Evolution of class B floral homeotic proteins: Obligate heterodimerization originated from homodimerization. Mol. Biol. Evol. 19, 587-596.

12. Tzeng, T.Y. and Yang, C.H. (2001) A MADS box gene from lily (Lilium longiflorum) is sufficient to generate dominant negative mutation by interacting with PISTILLATA (PI) in Arabidopsis thaliana. Plant Cell Physiol. 42, 1156-1168.

13. Adachi, Y., Mori, S., and Nakano, M. (2005) Agrobacterium-mediated production of transgenic plants in Tricyrtis hirta (Liliaceae). Acta. Hort. 673, 415-419.

14. Nakamura, T., Fukuda, T., Nakano, M., Hasebe, M., Kameya, T., and Kanno, A. (2005) The modified ABC model explains the development of the petaloid perianth of Agapanthus praecox ssp. orientalis (Agapanthaceae) flowers. Plant Mol. Biol. 58, 435-445.

15. Nakada, M., Komatsu, M., Ochiai, T., Ohtsu, K., Nakazono, M., Nishizawa, N.K., Nitta, K., Nishiyama, R., 
Kameya, T., and Kanno, A. (2006) Isolation of MaDEF from Muscari armeniacum and analysis of its expression using laser microdissection. Plant Sci. 170, 143-150.

16. Tsaftaris, A.S., Polidoros, A.N., Pasentsis, K., and Kalivas, A. (2006) Tepal formation and expression pattern of B-class paleoAP3-like MADS-box genes in crocus (Crocus sativus L.). Plant Sci. 170, 238-246.

17. Tsai, W.-C., Kuoh, C.-S., Chuang, M.-H., Chen, W.-H., and Chen, H.-H. (2004) Four DEF-like MADS box genes displayed distinct floral morphogenetic roles in Phalaenopsis orchid. Plant Cell Physiol. 45, 831-844.

18. Tsai, W.-C., Lee, P.-F., Chen, H.-I., Hsiao ,Y.-Y., Wei, W.-J., Pan, Z.-J., Chuang, M.-H., Kuoh, C.-S., Chen, W.-H., and Chen, H.-H. (2005) PeMADS6, a GLOBOSA/PISTILLATA-like gene in Phalaenopsis equestris involved in petaloid formation, and correlated with flower longevity and ovary development. Plant Cell Physiol. 46, 1125-1139.

19. Xu, Y., Teo, L.L. Zhou, J., Kumar, P.P., and Yu, H. (2006) Floral organ identity genes in the orchid Dendrobium crumenatum. Plant J. 46, 54-68.

20. Hsu, H.F. and Yang, C.H. (2002) An Orchid (Oncidium Gower Ramsey) AP3-like MADS gene regulates floral formation and initiation. Plant Cell Physiol. 43,1198-1209.

21. Park, J.H., Ishikawa, Y., Yoshida, R., Kanno, A., and Kameya, T. (2003) Expression of AODEF, a Bfunctional MADS-box gene, in stamens and inner tepals of dioecious species Asparagus officinalis L. Plant Mol. Biol. 51, 867-875.

22. Park, J.H., Ishikawa,Y., Ochiai, T., Kanno, A., and Kameya, T. (2004) Two GLOBOSA-like genes are expressed in second and third whorls of homochlamydeous flowers in Asparagus officinalis L. Plant Cell Physiol. 45, 325-332.

23. Kanno, A., Park, J.H., Ochiai, T., and Kameya, T. (2004) Floral organ identity genes involved in tepal development in asparagus. Flower. Newsl. 38, 10-18.

24. Asada, Y., Kasai, N., Adachi, Y., Kanno, A., Ito, N., Yun, P.-Y., and Masuda, K. (2006) A vegetative line of asparagus (Asparagus officinalis) with a homeotic change in flower development is correlated with functional deficiency in class-B MADS-box genes. J. Hort. Sci. Biotech. 81, 874-882.

25. Jack, T., Fox, G.L., and Meyerowitz, E.M. (1994) Arabidopsis homeotic gene APETALA3 ectopic expression: transcriptional and posttranscriptional regulation determines floral organ identity. Cell 76, 703716.

26. Krizek, B.A. and Meyerowitz, E.M. (1996) The Arabidopsis homeotic genes APETALA3 and PISTILLATA are sufficient to provide the B class organ identity function. Development 122, 11-22.

27. Davies, B., Rosa, A.D., Eneva, T., Saedler, H., and Sommer, H. (1996) Alteration of tobacco floral organ identity by expression of combinations of Antirrhinum MADS-box genes. Plant J. 10, 663-677.

28. Zahn, L.M., Leebens-Mack, J., dePamphilis, C.W., Ma, H., and Theissen, G. (2005) To be or not to B a flower: The role of DEFICIENS and GLOBOSA orthologs in the evolution of the angiosperms. J. Hered. 96, 225-240.

29. Jover-Gil, S., Candela, H., and Ponce M.-R. (2005) Plant microRNAs and development. Int. J. Dev. Biol. 49, 733-744.

30. Theissen, G. and Saedler, H., (2001) Floral quartets. Nature 409, 469-471.

31. Kramer, E.M., Stilio, V.S., and Schluter, P.M. (2003) Complex patterns of gene duplication in the APETALA3 and PISTILLATA lineages of the Rununculaceae. Int. J. Plant Sci. 164, 1-11.

32. Suzuki, S., Supaibulwatana, K., Mii, M., and Nakano, M. (2001) Production of transgenic plants of the Liliaceous ornamental plant Agapanthus pracox ssp. orientalis (Leighton) Leighton via Agrobacteriummediated transformation of embryogenic calli. Plant Sci. 161, 89-97.

33. Suzuki, S. and Nakano, M. (2002) Agrobacterium-mediated production of transgenic plants of Muscari armeniacum Leichtl. Ex Bak. Plant Cell Rep. 20, 835-841.

This article should be cited as follows:

Kanno, A., Nakada, M., Akita, Y., and Hirai, M. (2007) Class B gene expression and the modified ABC model in nongrass monocots. TSW Development \& Embryology 2, 17-28. DOI 10.1100/tswde.2007.37. 

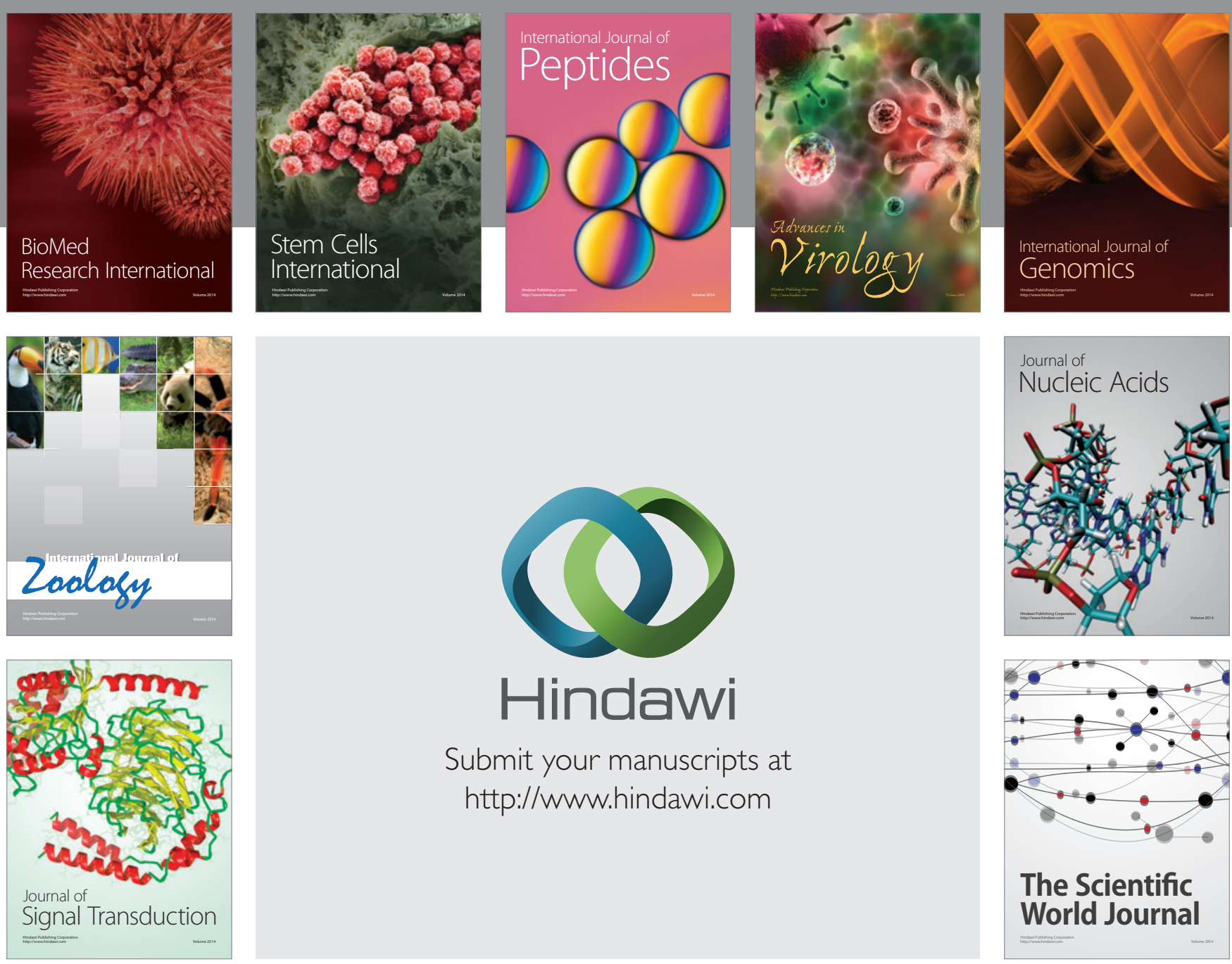

Submit your manuscripts at

http://www.hindawi.com
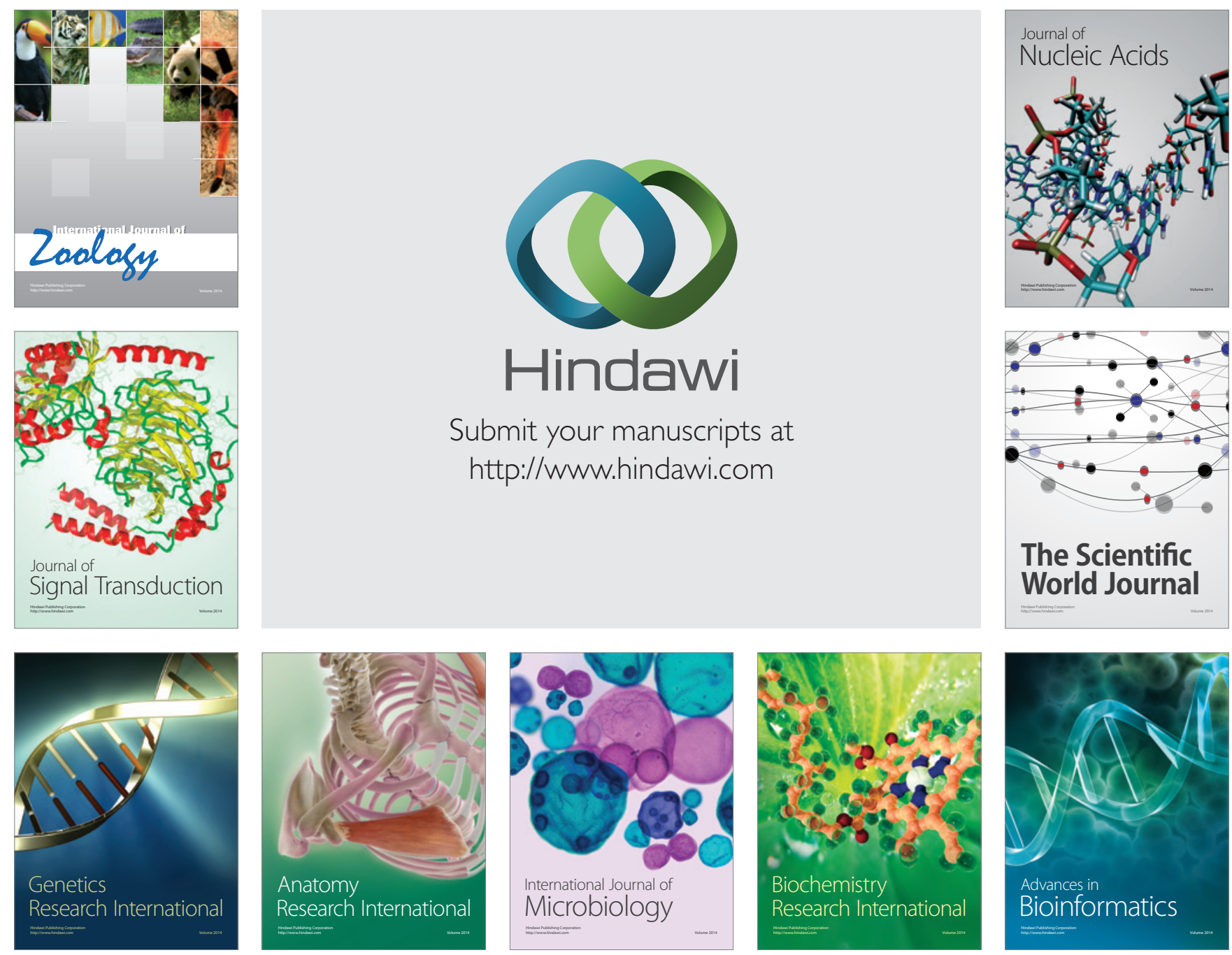

The Scientific World Journal
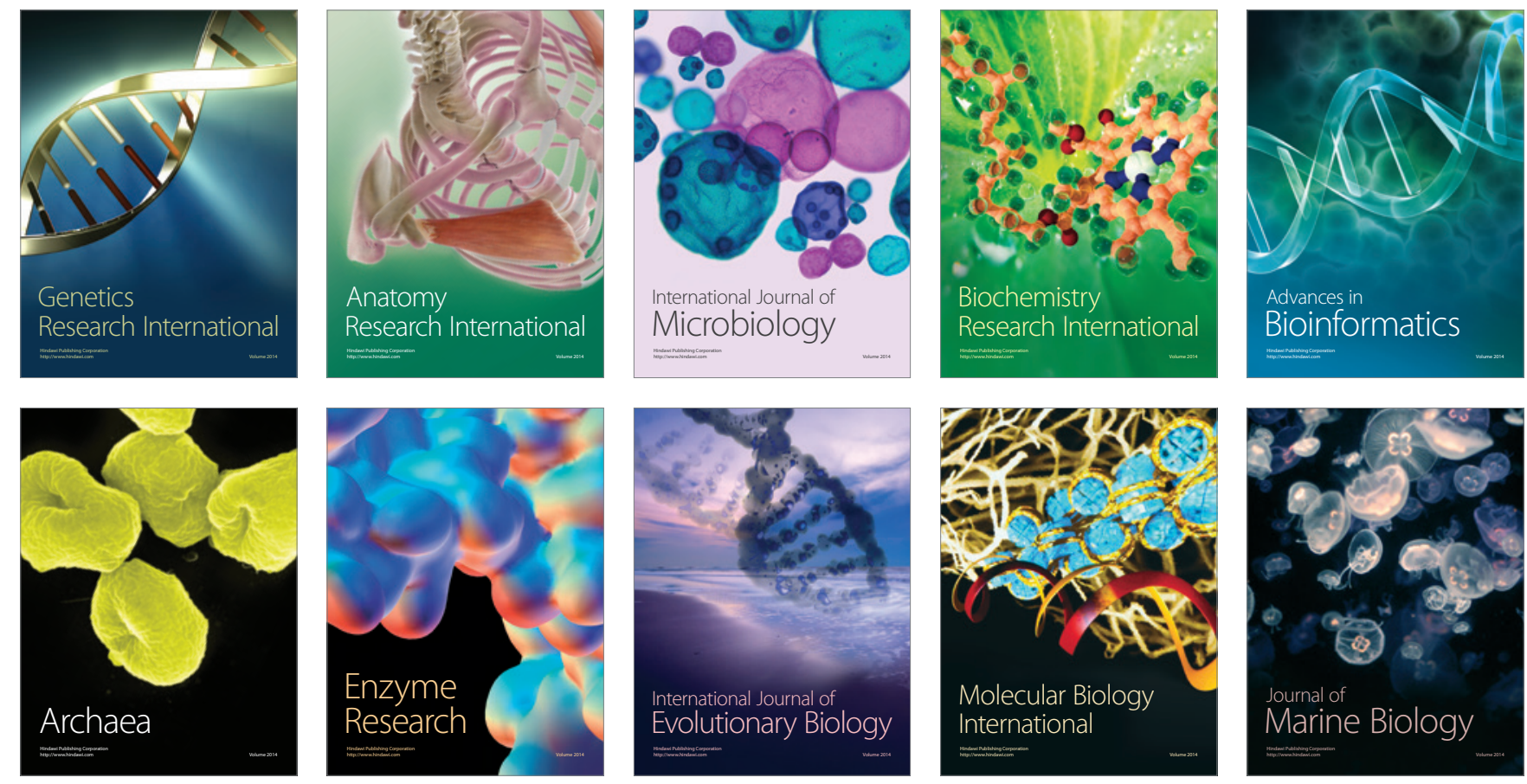